\title{
On Self-Similar Bernstein Functions and Corresponding Generalized Fractional Derivatives
}

\author{
Peter Kern ${ }^{1}$ (D) Svenja Lage ${ }^{1}$
}

Received: 17 June 2021 / Revised: 28 November 2021 / Accepted: 6 February 2022

(c) The Author(s) 2022

\begin{abstract}
We use the theory of Bernstein functions to analyze power law tail behavior with logperiodic perturbations which corresponds to self-similarity of the Bernstein functions. Such tail behavior appears in the context of semistable Lévy processes. The Bernstein approach enables us to solve some open questions concerning semi-fractional derivatives recently introduced in Kern et al. (Fract Calc Appl Anal 22(2):326-357, 2019) by means of the generators of certain semistable Lévy processes. In particular, it is shown that semi-fractional derivatives can be seen as generalized fractional derivatives in the sense of Kochubei (Integr Equ Oper Theory 71:583-600, 2011) and generalized fractional derivatives can be constructed by means of arbitrary Bernstein functions vanishing at the origin.
\end{abstract}

Keywords Power law tails · Log-periodic behavior - Laplace exponent - Bernstein functions · Self-similarity · Discrete scale invariance $\cdot$ Semistable Lévy process . Semi-fractional derivative $\cdot$ Semi-fractional diffusion $\cdot$ Sonine kernel $\cdot$ Sibuya distribution $\cdot$ Space-time duality

Mathematics Subject Classification (2020) Primary 26A33; Secondary 35R11 . 44A10 - 60E07 - 60G22 - 60G51 - 60G52

Dedicated to the memory of Mark Meerschaert (1955-2020).

$\bowtie$ Peter Kern

kern@hhu.de

Svenja Lage

Svenja.Lage@uni-duesseldorf.de

1 Mathematical Institute, Heinrich-Heine-University Düsseldorf, Universitätsstr. 1, 40225 Düsseldorf, Germany 


\section{Introduction}

A non-negative function $\widetilde{\psi}:(0, \infty) \rightarrow(0, \infty)$ is called a Bernstein function if it is of class $C^{\infty}(0, \infty)$ and

$$
(-1)^{n-1} \widetilde{\psi}^{(n)}(x) \geq 0 \text { for all } n \in \mathbb{N} \text { and } x>0 .
$$

Its first derivative $f=\widetilde{\psi}^{\prime}$ is a completely monotone function, i.e.

$$
(-1)^{n} f^{(n)}(x) \geq 0 \text { for all } n \in \mathbb{N}_{0} \text { and } x>0 .
$$

Due to a celebrated result of Bernstein, the completely monotone function $f$ is the Laplace transform of a unique Borel measure $\mu$ on $[0, \infty)$

$$
f(x)=\tilde{\mu}(x):=\int_{0}^{\infty} e^{-x t} \mathrm{~d} \mu(t) .
$$

As a consequence, the Bernstein function admits the representation

$$
\widetilde{\psi}(x)=a+b x+\int_{0}^{\infty}\left(1-e^{-x t}\right) \mathrm{d} \phi(t)
$$

for a unique triplet $[a, b, \phi]$, where $a, b \geq 0$ and $\phi$ is a Borel measure on $(0, \infty)$ satisfying $\int_{0}^{\infty} \min \{1, t\} \mathrm{d} \phi(t)<\infty$, also called the Lévy measure. For details on Bernstein functions, completely monotone functions and their connection to stochastic processes we refer to the monograph [31]. It is well known that in case $a=b=0$ the Bernstein function $\widetilde{\psi}(x)=\int_{0}^{\infty}\left(1-e^{-x t}\right) \mathrm{d} \phi(t)$ is the Laplace exponent of a Lévy subordinator $\left(X_{t}\right)_{t \geq 0}$, i.e. $\mathbb{E}\left[\exp \left(-s X_{t}\right)\right]=\exp (-t \cdot \widetilde{\psi}(s))$ for all $t \geq 0, s>0$, where $\left(X_{t}\right)_{t \geq 0}$ is a Lévy process with almost surely non-decreasing sample paths.

In Sect. 2 we will introduce a self-similarity property for Bernstein functions which is intimately connected to the following class of functions.

Definition 1.1 We call a function $\theta: \mathbb{R} \rightarrow \mathbb{R}$ admissible with respect to the parameters $\alpha \in(0,2) \backslash\{1\}$ and $c>1$ if the following three conditions are fulfilled:

$$
\begin{aligned}
& \theta(x)>0 \text { for all } x \in \mathbb{R}, \\
& \text { the mapping } t \mapsto t^{-\alpha} \theta(\log t) \text { is non-increasing for } t>0, \\
& \theta \text { is } \log \left(c^{1 / \alpha}\right) \text {-periodic. }
\end{aligned}
$$

We will frequently require that an admissible function $\theta$ is smooth, meaning that it is continuous and piecewise continuously differentiable.

In case $\alpha \in(0,1)$ we use an admissible function $\theta$ to define

$$
\phi(t, \infty):=t^{-\alpha} \theta(\log t) \text { for all } t>0
$$


as the positive tail of a Lévy measure $\phi$ concentrated on $(0, \infty)$ which belongs to a semistable distribution $v$ with $\log$-characteristic function

$$
\psi(x)=\int_{0}^{\infty}\left(e^{i x y}-1\right) \mathrm{d} \phi(y) \quad \text { for all } x \in \mathbb{R}
$$

given uniquely by the Fourier transform $\widehat{v}(x)=\int_{\mathbb{R}} e^{i x y} d \nu(y)=\exp (\psi(x))$. The corresponding Lévy process $\left(X_{t}\right)_{t \geq 0}$ given by $\mathbb{E}\left[\exp \left(i x \cdot X_{t}\right)\right]=\exp (t \cdot \psi(x))$ for all $t \geq 0$ and $x \in \mathbb{R}$ is called a semistable subordinator. For details on semistable distributions and Lévy processes we refer to the monographs [21,30]. The power law tail behavior with log-periodic perturbations of an admissible function $\theta$ naturally appears in various applications of natural sciences and other areas; see [34] and section 5.4 in [35]. In recent years the asymptotic fine structure of the corresponding measure $\phi$ and the function $\psi$ has drawn some attention; cf. [14, 15]. Given an admissible function $\theta$ with respect to $\alpha \in(0,1)$ and $c>1$ with corresponding semistable Lévy measure $\phi$ given by (1.8), by Definition 2.2 in [13] the semi-fractional derivative $\frac{\partial^{\alpha}}{\partial_{c, \theta} x^{\alpha}}$ of order $\alpha \in(0,1)$ is given by the non-local operator

$$
\frac{\partial^{\alpha}}{\partial_{c, \theta} x^{\alpha}} f(x):=-L f(x)=\int_{0}^{\infty}(f(x)-f(x-y)) \mathrm{d} \phi(y),
$$

where $L$ is the generator of the corresponding semistable Lévy process and at least functions $f$ in the Sobolev space $W^{2,1}(\mathbb{R})$ belong to the domain of the semi-fractional derivative. In terms of the Fourier transform we can equivalently rewrite (1.10) as

$$
\widehat{\frac{\partial^{\alpha}}{\partial_{c, \theta} x^{\alpha}}} f(x):=-\widehat{L f}(x)=-\psi(x) \widehat{f}(x) \text { for all } x \in \mathbb{R}
$$

where the Fourier transform of $f$ is given by $\widehat{f}(x)=\int_{\mathbb{R}} e^{i x y} f(y) d y$; see [13] for details. In Sect. 2, we will start with the elementary observation that for $\alpha \in(0,1)$ there is a one-to-one correspondence between self-similar Bernstein functions given as the Laplace exponent $\widetilde{\psi}(x)=-\psi(i x)$ for $x>0$ and semistable Lévy measures $\phi$ of the form (1.8). In particular, this enables us to show that semi-fractional derivatives of order $\alpha \in(0,1)$ can be seen as a special case of generalized fractional derivatives in the sense of [17]. A constant function $\theta$ corresponds to the complete Bernstein function $\widetilde{\psi}(x)=x^{\alpha}$ and an ordinary fractional derivative of order $\alpha \in(0,1)$. For details on classical fractional derivatives we refer to the monographs $[16,26,28]$. In fact we will show that the construction of generalized fractional derivatives is not restricted to self-similar Bernstein functions and holds for arbitrary Bernstein functions with $a=b=0$ in (1.4).

In Sect. 3 we will prove a discrete approximation formula of the generator in (1.10) involving a generalized Sibuya distribution given in terms of the self-similar Bernstein function.

In case $\alpha \in(1,2)$ we use an admissible function $\theta$ to define

$$
\phi(-\infty,-t):=t^{-\alpha} \theta(\log t) \text { for all } t>0
$$


as the negative tail of a Lévy measure $\phi$ concentrated on $(-\infty, 0)$ which belongs to a different semistable distribution $v$ with log-characteristic function

$$
\psi(x)=\int_{-\infty}^{0}\left(e^{i x y}-1-i x y\right) \mathrm{d} \phi(y) \quad \text { for all } x \in \mathbb{R} .
$$

Given an admissible function $\theta$ with respect to $\alpha \in(1,2)$ and $c>1$ with corresponding semistable Lévy measure $\phi$ given by (1.12), according to Definition 2.5 in [13] the negative semi-fractional derivative $\frac{\partial^{\alpha}}{\partial_{c, \theta}(-x)^{\alpha}}$ of order $\alpha \in(1,2)$ is given by the nonlocal operator

$$
\frac{\partial^{\alpha}}{\partial_{c, \theta}(-x)^{\alpha}} f(x):=L f(x)=\int_{0}^{\infty}\left(f(x+y)-f(x)-y f^{\prime}(x)\right) \mathrm{d} \phi(-y),
$$

where $L$ is again the generator of the corresponding semistable Lévy process and at least functions $f$ in the Sobolev space $W^{2,1}(\mathbb{R})$ belong to the domain of the semifractional derivative. For $\alpha \in(1,2)$ the function $x \mapsto \psi(-i x)$ cannot be a Bernstein function, but we will show in Sect. 4 that it has an inverse which is a self-similar Bernstein function. This will enable us to solve an open question from [12] concerning space-time duality for semi-fractional differential equations.

\section{Self-Similar Bernstein Functions and Semi-Fractional Derivatives}

In this section we suppose that $\alpha \in(0,1)$ in which case the Laplace exponent $\widetilde{\psi}$ of the corresponding semistable distribution $v$, uniquely given by the Laplace transform $\widetilde{v}(x)=\int_{0}^{\infty} e^{-x y} d v(y)=\exp (-\widetilde{\psi}(x))$ for $x>0$, in view of (1.9) can be represented as

$$
\widetilde{\psi}(x)=-\psi(i x)=\int_{0}^{\infty}\left(1-e^{-x y}\right) \mathrm{d} \phi(y) .
$$

Clearly, $\widetilde{\psi}$ is a Bernstein function since $\phi$ integrates $\min \{1, t\}$ on $(0, \infty)$.

Definition 2.1 We call a Bernstein function $\widetilde{\psi}$ self-similar with respect to $\alpha \in(0,1)$ and $c>1$ if it admits the discrete scale invariance

$$
\widetilde{\psi}\left(c^{1 / \alpha} x\right)=c \cdot \widetilde{\psi}(x) \text { for all } x>0
$$

Clearly, self-similarity can be iterated leading to $\widetilde{\psi}\left(c^{m / \alpha} x\right)=c^{m} \cdot \widetilde{\psi}(x)$ for all $x>0$ and $m \in \mathbb{Z}$. Moreover, the set of self-similarity factors

$$
\mathbb{S}=\left\{c>0: \widetilde{\psi}\left(c^{1 / \alpha} x\right)=c \cdot \widetilde{\psi}(x) \text { for all } x>0\right\}
$$

is a closed multiplicative subgroup of $(0, \infty)$ due to continuty of $\widetilde{\psi}$, such that we either have the discrete scale invariance $\mathbb{S}=c^{\mathbb{Z}}$ for some $c>1$, or continuous scaling $\mathbb{S}=(0, \infty)$. Our focus is on the discrete scale behavior and we will give an 
impression of how corresponding self-similar Bernstein functions look like after the following elementary observation, which is our key result.

Lemma 2.2 For fixed $\alpha \in(0,1)$ and $c>1$ the following statements are equivalent.

(i) $\widetilde{\psi}$ is a self-similar Bernstein function with respect to $\alpha \in(0,1)$ and $c>1$.

(ii) $\widetilde{\psi}(x)=\int_{0}^{\infty}\left(1-e^{-x y}\right) d \phi(y)$, where the Lévy measure $\phi$ is given by (1.12) for some admissible function $\theta$ with respect to $\alpha \in(0,1)$ and $c>1$.

In either case we have $\widetilde{\psi}(x)=x^{\alpha} \gamma(-\log x)$, where

$$
\gamma(x)=\int_{0}^{\infty} e^{-t} t^{-\alpha} \theta(\log t+x) d t
$$

is an admissible $C^{\infty}(\mathbb{R})$-function with respect to $\alpha \in(0,1)$ and $c>1$.

Proof “(i) $\Rightarrow$ (ii)": Since $\widetilde{\psi}$ is a Bernstein function, by (1.4) we have

$$
\widetilde{\psi}(x)=a+b x+\int_{0}^{\infty}\left(1-e^{-x t}\right) \mathrm{d} \phi(t)
$$

for some $a, b \geq 0$ and a Lévy measure $\phi$ on $(0, \infty)$ integrating $\min \{1, t\}$. Iterating the self-similarity relation shows that $\widetilde{\psi}\left(c^{m / \alpha} x\right)=c^{m} \widetilde{\psi}(x)$ for all $x>0$ and $m \in \mathbb{Z}$. As $m \rightarrow-\infty$, we see that $\lim _{x \downarrow 0} \widetilde{\psi}(x)=0$ and hence $a=0$. By the transformation rule self-similarity now reads as

$$
\begin{aligned}
\widetilde{\psi}\left(c^{1 / \alpha} x\right) & =b c^{1 / \alpha} x+\int_{0}^{\infty}\left(1-e^{-x t}\right) \mathrm{d}\left(c^{1 / \alpha} \phi\right)(t) \\
& =b c x+c \cdot \int_{0}^{\infty}\left(1-e^{-x t}\right) \mathrm{d} \phi(t)=c \cdot \widetilde{\psi}(x),
\end{aligned}
$$

where $\left(c^{1 / \alpha} \phi\right)$ denotes the image measure under scale multiplication with $c^{1 / \alpha}$. Since the Bernstein triplet $[a, b, \phi]$ is unique, we must have $b=0$ and $\left(c^{1 / \alpha} \phi\right)=c \cdot \phi$. Hence by Lemma 7.1.6 and Corollary 7.4.4 in [21] we have that $\phi$ is a $\left(c^{1 / \alpha}, c\right)$-semistable Lévy measure on $(0, \infty)$ fulfilling (1.12) for some admissible function $\theta$ with respect to $\alpha \in(0,1)$ and $c>1$. The connection of Corollary 7.4.4 in [21] to admissible functions is made precise by Lemma A.1 in the Appendix.

“(ii) $\Rightarrow$ (i)": Clearly, $\widetilde{\psi}$ is a Bernstein function by Theorem 3.2 in [31] and selfsimilarity follows from (2.4) with $b=0$ which is valid by Lemma 7.1.6 in [21].

Now define $\gamma(x):=e^{\alpha x} \widetilde{\psi}\left(e^{-x}\right)$ which is of class $C^{\infty}(\mathbb{R})$ by the product rule and $\gamma(x)>0$ for all $x \in \mathbb{R}$. Then $\gamma$ is $\log \left(c^{1 / \alpha}\right)$-periodic, since

$$
\gamma\left(x+\log \left(c^{1 / \alpha}\right)\right)=e^{\alpha x} c \cdot \widetilde{\psi}\left(c^{-1 / \alpha} e^{-x}\right)=e^{\alpha x} \widetilde{\psi}\left(e^{-x}\right)=\gamma(x),
$$

and $t \mapsto t^{-\alpha} \gamma(\log t)=\widetilde{\psi}\left(t^{-1}\right)$ is non-increasing. Hence $\gamma$ is an admissible function with respect to $\alpha \in(0,1)$ and $c>1$. Moreover, by partial integration and a change 
of variables $t=x y$ we get

$$
\begin{aligned}
\widetilde{\psi}(x) & =\int_{0}^{\infty}\left(1-e^{-x y}\right) \mathrm{d} \phi(y)=x \int_{0}^{\infty} e^{-x y} y^{-\alpha} \theta(\log y) \mathrm{d} y \\
& =x^{\alpha} \int_{0}^{\infty} e^{-t} t^{-\alpha} \theta(\log t-\log x) \mathrm{d} t=x^{\alpha} \gamma(-\log x),
\end{aligned}
$$

which shows that (2.3) holds, concluding the proof.

Example 2.3 Since $\gamma$ in (2.3) is admissible with respect to the same parameters $\alpha \in$ $(0,1)$ and $c>1$ as $\theta$, we may iterate the procedure. Starting with $\gamma_{0}:=\theta$ and setting

$$
\gamma_{k}(x):=\int_{0}^{\infty} e^{-t} t^{-\alpha} \gamma_{k-1}(\log t+x) \mathrm{d} t
$$

for $k \in \mathbb{N}$, we have $\gamma_{1}=\gamma$ and the sequence $\left(\widetilde{\psi}_{k}\right)_{k \in \mathbb{N}}$ given by $\widetilde{\psi}_{k}(x)=x^{\alpha} \gamma_{k}(-\log x)$ is a sequence of self-similar Bernstein functions by Lemma 2.2.

Example 2.4 If $\mathbb{S}=(0, \infty)$ in (2.2), then by Lemma 2.2 the function $\theta$ and hence also the function $\gamma$ must be constant. Thus $\widetilde{\psi}$ is the well known prototype of a Bernstein function $\widetilde{\psi}(x)=C x^{\alpha}$ for some $C>0$ and $\alpha \in(0,1)$. To give an explicit example with $\mathbb{S}=c^{\mathbb{Z}}$, for fixed $\alpha \in(0,1)$ we consider the simple periodic function $\theta(x)=$ $1+\frac{1}{\alpha}+\sin x$, which is positive and $\log \left(c^{1 / \alpha}\right)$-periodic for $c=e^{2 \pi \alpha}$. Moreover, $\theta$ is a smooth admissible function by Lemma A.1(v) in the Appendix, since $\theta^{\prime}(x)=$ $\cos x \leq 1+\alpha(1+\sin x)=\alpha \theta(x)$. For the corresponding function $\gamma$ we have

$$
\begin{aligned}
\gamma(x) & =\int_{0}^{\infty} e^{-t} t^{-\alpha}\left(1+\frac{1}{\alpha}+\sin (\log t+x)\right) \mathrm{d} t \\
& =\left(1+\frac{1}{\alpha}\right) \Gamma(1-\alpha)+\int_{0}^{\infty} e^{-t} t^{-\alpha} \sin (\log t+x) \mathrm{d} t
\end{aligned}
$$

and hence the self-similar Bernstein function has the representation

$$
\widetilde{\psi}(x)=x^{\alpha}\left(\left(1+\frac{1}{\alpha}\right) \Gamma(1-\alpha)+\int_{0}^{\infty} e^{-t} t^{-\alpha} \sin (\log t-\log x) \mathrm{d} t\right) .
$$

Now, for $c=e^{2 \pi \alpha}$ we have

$$
\begin{aligned}
\gamma\left(-\log \left(\sqrt{c}^{1 / \alpha} x\right)\right) & =\left(1+\frac{1}{\alpha}\right) \Gamma(1-\alpha)+\int_{0}^{\infty} e^{-t} t^{-\alpha} \sin (\log t-\log x-\pi) \mathrm{d} t \\
& =\left(1+\frac{1}{\alpha}\right) \Gamma(1-\alpha)-\int_{0}^{\infty} e^{-t} t^{-\alpha} \sin (\log t-\log x) \mathrm{d} t \\
& =\gamma(-\log x)-2 \int_{0}^{\infty} e^{-t} t^{-\alpha} \sin (\log t-\log x) \mathrm{d} t,
\end{aligned}
$$


which cannot coincide with $\gamma(-\log x)$ for all $x>0$. Hence $\widetilde{\psi}\left(\sqrt{c}^{1 / \alpha} x\right) \not \equiv \sqrt{c} \widetilde{\psi}(x)$ and we have $\mathbb{S}=c^{\mathbb{Z}}$. Iterating the procedure as in Example 2.3 gives us

$$
\gamma_{k}(x)=\left(1+\frac{1}{\alpha}\right)(\Gamma(1-\alpha))^{k}+f_{k}(x)
$$

for some non-constant and periodic function $f_{k}$ with $\left|f_{k}(x)\right| \leq(\Gamma(1-\alpha))^{k}$ for all $x \in \mathbb{R}$ and $k \in \mathbb{N}$. Since $\Gamma(1-\alpha)>1$ this shows that in general we cannot expect convergence of the sequence $\left(\widetilde{\psi}_{k}\right)_{k \in \mathbb{N}}$ in Example 2.3.

Remark 2.5 If we assume that the admissible function $\theta$ is smooth, then it admits a Fourier series representation

$$
\theta(x)=\sum_{k \in \mathbb{Z}} c_{k} e^{i k \tilde{c} x} \quad \text { with } \quad \tilde{c}=\frac{2 \pi \alpha}{\log c}
$$

In this case the function $\gamma$ appearing in Lemma 2.2 is given by the modified Fourier series

$$
\gamma(x)=\sum_{k \in \mathbb{Z}} c_{k} \Gamma(i k \tilde{c}-\alpha+1) e^{i k \tilde{c} x} \quad \text { for } x \in \mathbb{R}
$$

which can be seen as follows. By Theorem 3.1 in [13] the coefficients of this series appear in a representation of the log-characteristic function

$$
\psi(x)=-\sum_{k \in \mathbb{Z}} c_{k} \Gamma(i k \tilde{c}-\alpha+1)(-i x)^{\alpha-i k \tilde{c}} \quad \text { for } x \in \mathbb{R}
$$

and the relation $\gamma(x)=e^{\alpha x} \widetilde{\psi}\left(e^{-x}\right)=-e^{\alpha x} \psi\left(i e^{-x}\right)$ easily shows (2.6).

A natural question which arises is, if for two admissible functions $\theta_{1}, \theta_{2}$ with respect to the parameters $\alpha_{1} \in(0,1)$ and $c_{1}>1$, respectively $\alpha_{2} \in(0,1)$ and $c_{2}>1$, the composition of the corresponding semi-fractional derivatives given by (1.10) can again be a semi-fractional derivative of order $\alpha:=\alpha_{1}+\alpha_{2}$. We concentrate on the easiest case when $\alpha \in(0,1)$ and $\theta_{1}, \theta_{2}$ have the same periodicity, i.e. $c_{1}^{1 / \alpha_{1}}=c_{2}^{1 / \alpha_{2}}$. In view of (1.11) for the corresponding log-characteristic functions we need to show that

$$
\psi_{1}(x) \cdot \psi_{2}(x)=-\psi(x) \text { for all } x \in \mathbb{R},
$$

where $\psi$ is the log-characteristic function of a semistable distribution corresponding to an admissible function $\theta$ with respect to the parameters $\alpha \in(0,1)$ and $c:=c_{1}^{\alpha / \alpha_{1}}=$ $c_{2}^{\alpha / \alpha_{2}}>1$. Since (2.8) is equivalent to $\widetilde{\psi}_{1} \cdot \widetilde{\psi}_{2}=\widetilde{\psi}$ for the corresponding log-Laplace exponents and we have self-similarity

$$
\begin{aligned}
\widetilde{\psi}\left(c^{1 / \alpha} x\right) & =\widetilde{\psi}_{1}\left(c_{1}^{1 / \alpha_{1}} x\right) \cdot \widetilde{\psi}_{2}\left(c_{2}^{1 / \alpha_{2}} x\right)=c_{1} c_{2} \cdot \widetilde{\psi}_{1}(x) \widetilde{\psi}_{2}(x) \\
& =c^{\alpha_{1} / \alpha} c^{\alpha_{2} / \alpha} \cdot \widetilde{\psi}(x)=c \cdot \widetilde{\psi}(x),
\end{aligned}
$$

in view of Lemma 2.2 this is equivalent to require that $\widetilde{\psi}$ is a Bernstein function. 
Corollary $2.6 \widetilde{\psi}_{1} \cdot \widetilde{\psi}_{2}$ is a Bernstein function iff there exists an admissible function $\theta$ with respect to the parameters $\alpha=\alpha_{1}+\alpha_{2} \in(0,1)$ and $c=c_{1}^{\alpha / \alpha_{1}}=c_{2}^{\alpha / \alpha_{2}}>1$ such that

$$
\frac{\partial^{\alpha_{2}}}{\partial_{c_{2}, \theta_{2}} x^{\alpha_{2}}} \frac{\partial^{\alpha_{1}}}{\partial_{c_{1}, \theta_{1}} x^{\alpha_{1}}} f=\frac{\partial^{\alpha}}{\partial_{c, \theta} x^{\alpha}} f
$$

for suitable functions $f \in W^{2,1}(\mathbb{R})$ with $\frac{\partial^{\alpha_{1}}}{\partial_{c_{1}, \theta_{1}} x^{\alpha_{1}}} f$ belonging to the domain of $\frac{\partial^{\alpha_{2}}}{\partial_{c_{2}, \theta_{2}} x^{\alpha_{2}}}$.

Remark 2.7 In case $\theta_{1}, \theta_{2}$ are smooth admissible functions with Fourier series representations as in (2.5)

$$
\theta_{1}(x)=\sum_{k \in \mathbb{Z}} c_{k, 1} e^{i k \tilde{c} x} \quad \text { and } \quad \theta_{2}(x)=\sum_{\ell \in \mathbb{Z}} c_{\ell, 2} e^{i \ell \tilde{c} x},
$$

where $\tilde{c}=\frac{2 \pi \alpha_{1}}{\log c_{1}}=\frac{2 \pi \alpha_{2}}{\log c_{2}}=\frac{2 \pi \alpha}{\log c}$, then by (2.7) and the Cauchy product rule we easily get

$$
\tilde{\psi}(x)=\widetilde{\psi}_{1}(x) \cdot \widetilde{\psi}_{2}(x)=\sum_{m \in \mathbb{Z}} d_{m} \Gamma\left(i m \tilde{c}-\left(\alpha_{1}+\alpha_{2}\right)+1\right) x^{\alpha_{1}+\alpha_{2}-i m \tilde{c}},
$$

where

$$
d_{m}=\sum_{\ell \in \mathbb{Z}} c_{m-\ell, 1} c_{\ell, 2} \frac{\Gamma\left(i(m-\ell) \tilde{c}-\alpha_{1}+1\right) \Gamma\left(i \ell \tilde{c}-\alpha_{2}+1\right)}{\Gamma\left(i m \tilde{c}-\left(\alpha_{1}+\alpha_{2}\right)+1\right)} .
$$

Uniqueness of the Fourier coefficients then gives us the representation

$$
\theta(x)=\sum_{m \in \mathbb{Z}} d_{m} e^{i m \tilde{c} x}
$$

for the admissible function $\theta$ from Corollary 2.6, provided that (2.10) is a Bernstein function.

Finally, we want to show that the semi-fractional derivative of order $\alpha \in(0,1)$ can be seen as a special case of a generalized fractional derivative introduced in [17]. Starting with (1.10) for a smooth admissible function $\theta$, integration by parts shows that

$$
\frac{\partial^{\alpha}}{\partial_{c, \theta} x^{\alpha}} f(x)=\int_{0}^{\infty} f^{\prime}(x-y) y^{-\alpha} \theta(\log y) \mathrm{d} y
$$

as laid out in [13]. Introducing the kernel function $k(y):=y^{-\alpha} \theta(\log y)$ and restricting considerations to functions with support on the positive real line, this can be interpreted as a semi-fractional derivative of Caputo type

$$
\frac{\partial^{\alpha}}{\partial_{c, \theta} x^{\alpha}} f(x)=\int_{0}^{x} f^{\prime}(x-y) k(y) d y=\left(k * f^{\prime}\right)(x) .
$$


On the other hand, interchanging the order of integration and differentiation gives a semi-fractional derivative of Riemann-Liouville type

$$
\mathbb{D}_{c, \theta}^{\alpha} f(x)=\frac{\mathrm{d}}{\mathrm{d} x} \int_{0}^{x} f(x-y) k(y) \mathrm{d} y=(k * f)^{\prime}(x),
$$

which is also called of convolution type in [36]. The relationship between these forms is given by the formula

$$
\frac{\partial^{\alpha}}{\partial_{c, \theta} x^{\alpha}} f(x)=\mathbb{D}_{c, \theta}^{\alpha} f(x)-f(0) k(x)=: \mathbb{D}_{(k)} f(x)
$$

which can be derived as (2.33) in [22] and for more general kernel functions $\mathbb{D}_{(k)} f$ is called a generalized fractional derivative in [17]. For further approaches into this direction see $[3,18,20,25,29,36]$. Of particular interest are non-negative locally integrable kernel functions $k$ such that the operator $\mathbb{D}_{(k)}$ possesses a right inverse $\mathbb{I}_{(k)}$ such that $\mathbb{D}_{(k)} \mathbb{I}_{(k)} f=f$. Using the theory of complete Bernstein functions and the relationship to the Stieltjes class, it is shown in [17] that this is possible with

$$
\mathbb{I}_{(k)} f(x)=\int_{0}^{x} f(y) k^{*}(x-y) \mathrm{d} y
$$

for locally bounded measurable functions $f$ if $\left(k, k^{*}\right)$ forms a Sonine pair of kernels, i.e. $k * k^{*} \equiv 1$; cf. also $[27,33,37]$. In this case $\mathbb{I}_{(k)} f$ is called a generalized fractional integral of order $\alpha$ and it also holds that $\mathbb{I}_{(k)} \mathbb{D}_{(k)} f(x)=f(x)-f(0)$ for absolutely continuous functions $f$.

We will now show that a Sonine kernel $k^{*}$ may exist for our specific kernel function $k(y)=y^{-\alpha} \theta(\log y)$ working in the more general framework of Bernstein functions and their relation to completely monotone functions. As argued in [9], necessarily the kernel function $k$ should have an integrable singularity at the origin to be a meaningful generalization of a fractional derivative, but we are not going into this discussion. Hence we aim for possible extensions of the list of specific kernel functions given in section 6 of [20]. Our approach works similar to the method laid out in [17] but, to work in full generality, we have to relax the definition of a Sonine pair in the following sense.

Definition 2.8 Given two Borel measures $\eta, \rho$ on $[0, \infty)$ we say that $(\eta, \rho)$ forms a generalized Sonine pair if $\eta * \rho=\lambda$, where $\lambda$ denotes Lebesgue measure restricted to $[0, \infty)$.

Theorem 2.9 Let $\widetilde{\psi}$ be a Bernstein function with triplet $[0,0, \phi]$ and define $\eta$ as the measure on $[0, \infty)$ with $\eta(\{0\})=0$ and density $t \mapsto \phi(t, \infty)$ with respect to Lebesgue measure on $(0, \infty)$, i.e. $d \eta(t)=\phi(t, \infty) d t$. Then $\eta$ is a Borel measure and there exists another Borel measure $\rho$ such that $(\eta, \rho)$ forms a generalized Sonine pair. 
Proof First note that $\eta$ is locally finite, since by the Fubini-Tonelli theorem for all $y>0$ we have

$$
\eta([0, y])=\int_{0}^{y} \int_{t}^{\infty} \mathrm{d} \phi(x) \mathrm{d} t=\int_{0}^{\infty} \min \{x, y\} \mathrm{d} \phi(x)<\infty .
$$

Hence $\eta$ is a Borel measure. We have that $\widetilde{\psi}(x)=\int_{0}^{\infty}\left(1-e^{-x t}\right) d \phi(t)$ is a Bernstein function and thus $G(x):=\frac{1}{x} \widetilde{\psi}(x)$ is completely monotone by Corollary 3.8(iv) in [31]. The tail function of the Lévy measure is a right-continuous, non-increasing function and integration by parts yields the Laplace transform

$$
G(x)=\int_{0}^{\infty} \frac{1}{x}\left(1-e^{-x t}\right) \mathrm{d} \phi(t)=\int_{0}^{\infty} e^{-x t} \phi(t, \infty) \mathrm{d} t=\tilde{\eta}(x)
$$

for $x>0$. Further, by Theorem 3.7 in [31], $G^{*}(x):=1 / \widetilde{\psi}(x)$ is completely monotone, since it is the composition of a Bernstein function and the completely monotone function $x \mapsto \frac{1}{x}$. Hence there exists a Borel measure $\rho$ on $[0, \infty)$ such that $G^{*}(x)=$ $\int_{0}^{\infty} e^{-x t} d \rho(t)=\widetilde{\rho}(x)$, which serves as the generalized Sonine partner of $\eta$ as follows. We easily calculate the Laplace transform of $\eta * \rho$ as

$$
\widetilde{\eta}(x) \cdot \widetilde{\rho}(x)=G(x) \cdot G^{*}(x)=\frac{\widetilde{\psi}(x)}{x} \cdot \frac{1}{\widetilde{\psi}(x)}=\frac{1}{x}
$$

for $x>0$, which is the Laplace transform of the Lebesgue measure $\lambda$ on $[0, \infty)$. Now the assertion directly follows from uniqueness of the Laplace transform for probability measures and the translation principle; see Theorem 1a in chapter XIII.1 of [7].

By virtue of Theorem 2.9 we now define a generalized fractional derivative and a corresponding generalized fractional integral of suitable functions $f:[0, \infty) \rightarrow \mathbb{R}$ as

$$
\mathbb{D}_{(\eta)} f(x)=\int_{0}^{x} f^{\prime}(x-y) \mathrm{d} \eta(y)=\left(f^{\prime} * \eta\right)(x)
$$

for absolutely continuous functions $f$ with locally bounded density $f^{\prime}$, respectively

$$
\mathbb{I}_{(\rho)} f(x)=\int_{0}^{x} f(x-y) \mathrm{d} \rho(y)=(f * \rho)(x)
$$

for locally bounded measurable functions $f$.

Lemma 2.10 For absolutely continuous functions $f:[0, \infty) \rightarrow \mathbb{R}$ with locally bounded density $f^{\prime}$ we have

$$
\begin{aligned}
\mathbb{D}_{(\eta)} f(x) & =\frac{d}{d x} \int_{0}^{x} f(x-y) d \eta(y)-f(0) \phi(x, \infty) \\
& =(f * \eta)^{\prime}(x)-f(0) \phi(x, \infty)
\end{aligned}
$$

for almost all $x>0$. 
We may also use (2.16) as the definition of the generalized fractional derivative which has some advantage, since it can be defined for the broader class of locally bounded measurable functions $f$ due to $\int_{0}^{x} f(x-y) \mathrm{d} \eta(y)=\int_{0}^{x} f(x-y) \phi(y, \infty) d y$ being absolutely continuous.

Proof Let $k(x)=\phi(x, \infty)$ be the tail function of the Lévy measure. Then by partial integration and the Fubini-Tonelli theorem the Laplace transform on the right-hand side of (2.16) can be written as

$$
\begin{aligned}
& \int_{0}^{\infty} e^{-s x}(f * k)^{\prime}(x) \mathrm{d} x-f(0) \tilde{k}(s)=s \int_{0}^{\infty} e^{-s x}(f * k)(x) \mathrm{d} x-f(0) \tilde{k}(s) \\
& \quad=s \int_{0}^{\infty} e^{-s x} \int_{0}^{x} f(x-y) k(y) \mathrm{d} y \mathrm{~d} x-f(0) \tilde{k}(s) \\
& \quad=s \int_{0}^{\infty} k(y) \int_{y}^{\infty} e^{-s x} f(x-y) \mathrm{d} x \mathrm{~d} y-f(0) \tilde{k}(s) \\
& =s \tilde{f}(s) \int_{0}^{\infty} e^{-s y} k(y) \mathrm{d} y-f(0) \tilde{k}(s) \\
& =s \tilde{f}(s) \tilde{k}(s)-f(0) \tilde{k}(s)
\end{aligned}
$$

On the other hand, by the Fubini-Tonelli theorem and partial integration the Laplace transform of (2.14) is given by

$$
\begin{aligned}
& \int_{0}^{\infty} e^{-s x} \int_{0}^{x} f^{\prime}(x-y) k(y) \mathrm{d} y \mathrm{~d} x=\int_{0}^{\infty} k(y) \int_{y}^{\infty} e^{-s x} f^{\prime}(x-y) \mathrm{d} x \mathrm{~d} y \\
& =\int_{0}^{\infty} k(y)\left(-e^{-s y} f(0)+s \int_{y}^{\infty} e^{-s x} f(x-y) \mathrm{d} x\right) \mathrm{d} y \\
& =-f(0) \tilde{k}(s)+s \tilde{f}(s) \tilde{k}(s),
\end{aligned}
$$

which proves the assertion.

Theorem 2.11 Let the assumptions of Theorem 2.9 hold.

(a) For absolutely continuous functions $f$ with locally bounded density $f^{\prime}$ we have $\mathbb{I}_{(\rho)} \mathbb{D}_{(\eta)} f(x)=f(x)-f(0)$ for all $x>0$.

(b) For locally bounded measurable functions $f$ we have $\mathbb{D}_{(\eta)} \mathbb{I}_{(\rho)} f(x)=f(x)$ for almost all $x>0$. If $f$ is continuous then the relation holds for all $x>0$.

Proof (a) For absolutely continuous functions $f$ with density $f^{\prime}$ we get

$$
\begin{aligned}
\mathbb{I}_{(\rho)} \mathbb{D}_{(\eta)} f(x) & \left.=\int_{0}^{x} \mathbb{D}_{(\eta)} f(x-y)\right) \mathrm{d} \rho(y)=\int_{0}^{x}\left(f^{\prime} * \eta\right)(x-y) \mathrm{d} \rho(y) \\
& =\left(\left(f^{\prime} * \eta * \rho\right)(x)=\left(f^{\prime} * \lambda\right)(x)=\int_{0}^{x} f^{\prime}(x-t) \mathrm{d} t\right. \\
& =\int_{0}^{x} f^{\prime}(t) \mathrm{d} t=f(x)-f(0) .
\end{aligned}
$$


(b) Since $\mathbb{I}_{(\rho)} f(0)=0$ and $\int_{0}^{x} f(t) \mathrm{d} t$ is absolutely continuous, we get

$$
\begin{aligned}
\mathbb{D}_{(\eta)} \mathbb{I}_{(\rho)} f(x) & =\frac{\mathrm{d}}{\mathrm{d} x} \int_{0}^{x} \mathbb{I}_{(\rho)} f(x-y) \mathrm{d} \eta(y)=\frac{\mathrm{d}}{\mathrm{d} x}\left(\mathbb{I}_{(\rho)} f * \eta\right)(x) \\
& =\frac{\mathrm{d}}{\mathrm{d} x}(f * \rho * \eta)(x)=\frac{\mathrm{d}}{\mathrm{d} x}(f * \lambda)(x)=\frac{\mathrm{d}}{\mathrm{d} x} \int_{0}^{x} f(x-t) \mathrm{d} t \\
& =\frac{\mathrm{d}}{\mathrm{d} x} \int_{0}^{x} f(t) \mathrm{d} t=f(x)
\end{aligned}
$$

for almost all $x>0$. If $f$ is continuous, this relation holds for all $x>0$, since the last equality holds by the fundamental theorem of calculus.

In our specific context of self-similar Bernstein functions, the semi-fractional integral given by the Sonine partner $\rho$ in $(2.15)$ is also determined by a self-similar Bernstein function as follows.

Lemma 2.12 Let $\widetilde{\psi}$ be a self-similar Bernstein function with respect to $\alpha \in(0,1)$ and $c>1$. Let $\rho$ be the associated Sonine partner from above, then the primitive $G_{I}^{*}(x):=\int_{0}^{x} G^{*}(y) d y=\int_{0}^{x} \tilde{\rho}(y) d y$ is a self-similar Bernstein function with respect to $1-\alpha \in(0,1)$ and $d:=c^{\frac{1-\alpha}{\alpha}}>1$.

Proof From Lemma 2.2 we get the scaling relation

$$
G^{*}\left(c^{1 / \alpha} x\right)=\frac{1}{\widetilde{\psi}\left(c^{1 / \alpha} x\right)}=\frac{1}{c \cdot \widetilde{\psi}(x)}=c^{-1} G^{*}(x) \text { for all } x>0,
$$

which implies $\left(c^{1 / \alpha} \rho\right)=c^{-1} \cdot \rho$ for the measure $\rho$ in Theorem 2.9. In particular it follows that $\rho(\{0\})=0$ and by the Fubini-Tonelli theorem we get

$$
\begin{aligned}
G_{I}^{*}(x) & =\int_{0}^{x} G^{*}(y) \mathrm{d} y=\int_{0}^{x} \int_{0+}^{\infty} e^{-y t} \mathrm{~d} \rho(t) \mathrm{d} y \\
& =\int_{0+}^{\infty} \frac{1-e^{-x t}}{t} \mathrm{~d} \rho(t)=\int_{0}^{\infty}\left(1-e^{-x t}\right) \mathrm{d} \mu(t),
\end{aligned}
$$

where the Borel measure $\mu$ on $(0, \infty)$ is given by $\mathrm{d} \mu(t):=\frac{1}{t} \mathrm{~d} \rho(t)$ and integrates $\min \{1, t\}$ as shown in the proof of Theorem 3.2 in [31]; cf. also Proposition 3.5 in [31]. Thus the primitive $G_{I}^{*}(x)$ is a Bernstein function and the scaling relation gives us

$$
\begin{aligned}
G_{I}^{*}\left(c^{1 / \alpha} x\right) & :=\int_{0}^{\infty}\left(1-e^{-x c^{1 / \alpha} t}\right) \mathrm{d} \mu(t)=c^{1 / \alpha} \int_{0+}^{\infty} \frac{1-e^{-x c^{1 / \alpha} t}}{c^{1 / \alpha} t} \mathrm{~d} \rho(t) \\
& =c^{1 / \alpha} \int_{0+}^{\infty} \frac{1-e^{-x t}}{t} d\left(c^{1 / \alpha} \rho\right)(t) \\
& =c^{\frac{1}{\alpha}-1} \int_{0}^{\infty}\left(1-e^{-x t}\right) \mathrm{d} \mu(t)=c^{\frac{1-\alpha}{\alpha}} G_{I}^{*}(x) .
\end{aligned}
$$


Now let $d=c^{\frac{1-\alpha}{\alpha}}>1$ then $d^{\frac{1}{1-\alpha}}=c^{1 / \alpha}$ and we have $G_{I}^{*}\left(d^{\frac{1}{1-\alpha}} x\right)=d \cdot G_{I}^{*}(x)$ for all $x>0$. Hence $G_{I}^{*}$ is a self-similar Bernstein function with respect to $1-\alpha \in(0,1)$ and $d>1$.

Remark 2.13 By Lemma 2.2 the Lévy measure $\mu$ corresponding to $G_{I}^{*}$ is given by $\mu(t, \infty)=t^{\alpha-1} \sigma(\log t)$ for an admissible function $\sigma$ with respect to the parameters $1-\alpha \in(0,1)$ and $d>1$. If this admissible function $\sigma$ is smooth, we get a Sonine pair $\left(k, k^{*}\right)$ in the original sense as follows. Integration by parts yields

$$
G_{I}^{*}(x)=x \int_{0}^{\infty} \frac{1}{x}\left(1-e^{-x t}\right) \mathrm{d} \mu(t)=x \int_{0}^{\infty} e^{-x t} t^{\alpha-1} \sigma(\log t) \mathrm{d} t
$$

and hence we get as the derivative

$$
\begin{aligned}
G^{*}(x) & =\int_{0}^{\infty} e^{-x t} t^{\alpha-1} \sigma(\log t) \mathrm{d} t-x \int_{0}^{\infty} e^{-x t} t^{\alpha} \sigma(\log t) \mathrm{d} t \\
& =\int_{0}^{\infty} e^{-x t}\left(t^{\alpha-1} \sigma(\log t)-\frac{\mathrm{d}}{\mathrm{d} t}\left(t^{\alpha} \sigma(\log t)\right)\right) \mathrm{d} t \\
& =\int_{0}^{\infty} e^{-x t} t^{\alpha-1}\left((1-\alpha) \sigma(\log t)-\sigma^{\prime}(\log t)\right) \mathrm{d} t \\
& =: \int_{0}^{\infty} e^{-x t} k^{*}(t) \mathrm{d} t
\end{aligned}
$$

where the kernel $k^{*}$ is non-negative by Lemma A.1. Now the calculation of the Laplace transform in the proof of Theorem 2.9 shows that $\left(k, k^{*}\right)$ is a Sonine pair. Note that in general we cannot expect $k^{*}$ to be completely monotone as in the approach of [17] with complete Bernstein functions. Nor can we expect that the admissible function $\sigma$ is smooth in general.

\section{Discrete Approximation of the Generator}

Recall that by (1.10) the semi-fractional derivative operator of order $\alpha \in(0,1)$ is given by the negative generator of the continuous convolution semigroup $\left(v^{* t}\right)_{t \geq 0}$, where $v$ is the semistable distribution with log-characteristic function (1.9). If the tail of the Lévy measure is given by $\phi(t, \infty)=\frac{1}{\Gamma(1-\alpha)} t^{-\alpha}$, i.e. the admissible function $\theta \equiv \frac{1}{\Gamma(1-\alpha)}$ is constant, it is well known that the semi-fractional derivative coincides with the ordinary Riemann-Liouville fractional derivative of order $\alpha \in(0,1)$ and can be approximated by means of the Grünwald-Letnikov formula

$$
\frac{\partial^{\alpha}}{\partial x^{\alpha}} f(x)=\lim _{h \downarrow 0} h^{-\alpha} \sum_{j=0}^{\infty}\left(\begin{array}{l}
\alpha \\
j
\end{array}\right)(-1)^{j} f(x-j h)
$$

for functions $f$ in the Sobolev space $W^{2,1}(\mathbb{R})$; see section 2.1 in [22] for details. The coefficients in the Grünwald-Letnikov approximation formula appear in a discrete 
distribution on the positive integers called Sibuya distribution, which first appeared in [32]. A discrete random variable $X_{\alpha}$ on $\mathbb{N}$ is Sibuya distributed with parameter $\alpha \in(0,1)$ if

$$
\mathbb{P}\left(X_{\alpha}=j\right)=(-1)^{j-1}\left(\begin{array}{c}
\alpha \\
j
\end{array}\right)=(-1)^{j-1} \frac{\alpha(\alpha-1) \cdots(\alpha-j+1)}{j !} \text { for } j \in \mathbb{N} .
$$

For further details and extensions of the Sibuya distribution we refer to $[5,6,19]$ and the literature mentioned therein. Using (3.2) we may rewrite (3.1) as

$$
\begin{aligned}
\frac{\partial^{\alpha}}{\partial x^{\alpha}} f(x) & =\lim _{h \downarrow 0} h^{-\alpha} \sum_{j=0}^{\infty}\left(\begin{array}{c}
\alpha \\
j
\end{array}\right)(-1)^{j} f(x-j h) \\
& =\lim _{h \downarrow 0} h^{-\alpha}\left(f(x)-\sum_{j=1}^{\infty} \mathbb{P}\left(X_{\alpha}=j\right) f(x-j h)\right) \\
& =\lim _{h \downarrow 0} h^{-\alpha}\left(f(x)-\int_{\mathbb{R}} f(x-h y) d \mathbb{P}_{X_{\alpha}}(y)\right) \\
& =\lim _{h \downarrow 0} h^{-\alpha}\left(f *\left(\varepsilon_{0}-\mathbb{P}_{h X_{\alpha}}\right)\right)(x)
\end{aligned}
$$

which shows that the Grünwald-Letnikov formula is in fact a discrete approximation of the generator. For further relations of the Sibuya distribution to fractional diffusion equations see [23, 24].

Our aim is to generalize this formula for semi-fractional derivatives by means of the corresponding self-similar Bernstein functions. Note that for the Bernstein function $\widetilde{\psi}(x)=x^{\alpha}$ the nominator on the right-hand side of $(3.2)$ is given by $\widetilde{\psi}^{(j)}(1)$ and hence we may define a semi-fractional Sibuya distribution in the following way.

Definition 3.1 Given an admissible function $\theta$ with respect to $\alpha \in(0,1)$ and $c>1$, a semi-fractional Sibuya distributed random variable $X_{\theta}$ on $\mathbb{N}$ is given by

$$
\mathbb{P}\left(X_{\theta}=j\right)=\frac{(-1)^{j-1}}{j !} \frac{\widetilde{\psi}^{(j)}(1)}{\widetilde{\psi}(1)} \quad \text { for } j \in \mathbb{N}
$$

where $\widetilde{\psi}$ is the corresponding self-similar Bernstein function.

Clearly, the expression in (3.3) is non-negative by (1.1). Moreover, by monotone convergence and a Taylor series approach justified by Proposition 3.6 in [31] we get

$$
\begin{aligned}
\sum_{j=1}^{\infty} \frac{(-1)^{j-1}}{j !} \frac{\widetilde{\psi}^{(j)}(1)}{\widetilde{\psi}(1)} & =\frac{1}{\widetilde{\psi}(1)} \sum_{j=1}^{\infty} \frac{\widetilde{\psi}^{(j)}(1)}{j !} \lim _{\varepsilon \downarrow 0}(\varepsilon-1)^{j-1} \\
& =\frac{1}{\widetilde{\psi}(1)} \lim _{\varepsilon \downarrow 0} \frac{1}{\varepsilon-1}\left(\sum_{j=0}^{\infty} \frac{\widetilde{\psi}^{(j)}(1)}{j !}(\varepsilon-1)^{j}-\widetilde{\psi}(1)\right)
\end{aligned}
$$




$$
=\frac{1}{\widetilde{\psi}(1)} \lim _{\varepsilon \downarrow 0} \frac{1}{\varepsilon-1}(\widetilde{\psi}(\varepsilon)-\widetilde{\psi}(1))=\frac{\widetilde{\psi}(1)-\widetilde{\psi}(0)}{\widetilde{\psi}(1)}=1 .
$$

This shows that indeed (3.3) defines a proper distribution on $\mathbb{N}$ with pgf

$$
\begin{aligned}
G(z) & =\sum_{j=1}^{\infty} \mathbb{P}\left(X_{\theta}=j\right) z^{j}=-\frac{1}{\widetilde{\psi}(1)} \sum_{j=1}^{\infty} \frac{\widetilde{\psi}^{(j)}(1)}{j !}(-z)^{j} \\
& =1-\frac{1}{\widetilde{\psi}(1)} \sum_{j=0}^{\infty} \frac{\widetilde{\psi}^{(j)}(1)}{j !}((1-z)-1)^{j}=1-\frac{\widetilde{\psi}(1-z)}{\widetilde{\psi}(1)}
\end{aligned}
$$

for $|z| \leq 1$. Note that in the above arguments self-similarity of the Bernstein function is not needed. Hence (3.3) defines a proper distribution on $\mathbb{N}$ for every Bernstein function $\widetilde{\psi}$ with $\widetilde{\psi}(0)=0$.

Now let $\theta$ be a smooth admissible function having Fourier series representation

$$
\theta(x)=\sum_{k=-\infty}^{\infty} c_{k} e^{i k \tilde{c} x} \quad \text { with } \quad \tilde{c}=\frac{2 \pi \alpha}{\log c} .
$$

Then by Theorem 3.1 in [13] the corresponding self-similar Bernstein function can be expressed in terms of the log-characteristic function $\psi$ as

$$
\tilde{\psi}(x)=-\psi(i x)=\sum_{k=-\infty}^{\infty} \omega_{k} x^{\alpha-i k \tilde{c}} \quad \text { with } \quad \omega_{k}=c_{k} \Gamma(i k \tilde{c}-\alpha+1)
$$

and hence for $j \in \mathbb{N}_{0}$ we have

$$
\frac{\widetilde{\psi}^{(j)}(1)}{j !}=\sum_{k=-\infty}^{\infty} \omega_{k}\left(\begin{array}{c}
\alpha-i k \tilde{c} \\
j
\end{array}\right) .
$$

Note that these coefficients appear in a Grünwald-Letnikov-type approximation of the semi-fractional derivative given in Theorem 4.1 of [13] which enables us to prove the following approximation formula.

Theorem 3.2 Let $\theta$ be a smooth admissible function with respect to $\alpha \in(0,1)$ and $c>1$ with corresponding self-similar Bernstein function $\widetilde{\psi}$ and semi-fractional Sibuya distributed random variable $X_{\theta}$ given by (3.3). Then for $f \in W^{2,1}(\mathbb{R})$ the semifractional derivative can be approximated along the subsequence $h_{m}=c^{-m / \alpha}$ by

$$
\frac{\partial^{\alpha}}{\partial_{c, \theta} x^{\alpha}} f(x)=\lim _{m \rightarrow \infty} \widetilde{\psi}\left(h_{m}^{-1}\right)\left(f *\left(\varepsilon_{0}-\mathbb{P}_{h_{m} X_{\theta}}\right)\right)(x) .
$$


Proof First note that $h_{m}^{i k \tilde{c}}=c^{-i m k \tilde{c} / \alpha}=e^{-2 \pi i m k}=1$ for $\tilde{c}=\frac{2 \pi \alpha}{\log c}$ and by selfsimilarity we have $\widetilde{\psi}\left(h_{m}^{-1}\right)=h_{m}^{-\alpha} \widetilde{\psi}(1)$ for all $m \in \mathbb{N}$. Hence we get

$$
\begin{aligned}
& \tilde{\psi}\left(h_{m}^{-1}\right)\left(f *\left(\varepsilon_{0}-\mathbb{P}_{h_{m} X_{\theta}}\right)\right)(x)=h_{m}^{-\alpha} \widetilde{\psi}(1)\left(f(x)-\int_{\mathbb{R}} f\left(x-h_{m} y\right) d \mathbb{P}_{X_{\theta}}(y)\right) \\
& =h_{m}^{-\alpha} \widetilde{\psi}(1)\left(f(x)-\sum_{j=1}^{\infty} \frac{(-1)^{j-1}}{j !} \frac{\widetilde{\psi}^{(j)}(1)}{\widetilde{\psi}(1)} f\left(x-j h_{m}\right)\right) \\
& =h_{m}^{-\alpha}\left(\sum_{k=-\infty}^{\infty} \omega_{k} f(x)+\sum_{j=1}^{\infty}(-1)^{j} \sum_{k=-\infty}^{\infty} \omega_{k}\left(\begin{array}{c}
\alpha-i k \tilde{c} \\
j
\end{array}\right) f\left(x-j h_{m}\right)\right) \\
& \quad=h_{m}^{-\alpha} \sum_{j=0}^{\infty}(-1)^{j} \sum_{k=-\infty}^{\infty} \omega_{k} h_{m}^{i k \tilde{c}}\left(\begin{array}{c}
\alpha-i k \tilde{c} \\
j
\end{array}\right) f\left(x-j h_{m}\right) \rightarrow \frac{\partial^{\alpha}}{\partial_{c, \theta} x^{\alpha}} f(x),
\end{aligned}
$$

where the last convergence follows from Theorem 4.1 in [13].

\section{Space-Time Duality for Semi-Fractional Diffusions}

We first give a sufficient condition for an inverse function to be a Bernstein function.

Lemma 4.1 Let $f:(0, \infty) \rightarrow(0, \infty)$ be a $C^{\infty}(0, \infty)$-function such that $f^{\prime}$ is a Bernstein function and $f^{(n)}(x) \neq 0$ for all $x>0$ and $n \in \mathbb{N}$. Then its inverse $f^{-1}$ is a Bernstein function with $\left(f^{-1}\right)^{(n)}(x) \neq 0$ for all $x>0$ and $n \in \mathbb{N}$.

Proof We know that $f^{-1}(x)>0$ and $\left(f^{-1}\right)^{\prime}(x)=\frac{1}{f^{\prime}\left(f^{-1}(x)\right)}>0$ for all $x>0$. Moreover, as in Remark A.3 of the Appendix $\left(f^{-1}\right)^{\prime \prime}(x)=-\frac{f^{\prime \prime}\left(f^{-1}(x)\right)}{\left(f^{\prime}\left(f^{-1}(x)\right)\right)^{3}}<0$ for all $x>0$. For $n \geq 3$ we inductively use the formula for $\left(f^{-1}\right)^{(n)}$ given in Lemma A.2 of the Appendix. By induction we have

$$
\operatorname{sign}\left(\prod_{j=1}^{n-1}\left(\frac{\left(f^{-1}\right)^{(j)}(x)}{j !}\right)^{k_{j}}\right)=\prod_{j=1}^{n-1}(-1)^{(j-1) k_{j}}=(-1)^{\sum_{j=1}^{n-1}(j-1) k_{j}}
$$

and $\operatorname{sign}\left(f^{\left(k_{1}+\cdots+k_{n-1}\right)}\left(f^{-1}(x)\right)\right)=(-1)^{k_{1}+\cdots+k_{n-1}}$ since $k_{1}+\cdots+k_{n-1} \geq 2$ for $n \geq 3$ as $k_{1}+2 k_{2} \cdots+(n-1) k_{n-1}=n$. This shows that

$$
\operatorname{sign}\left(f^{\left(k_{1}+\cdots+k_{n-1}\right)}\left(f^{-1}(x)\right) \prod_{j=1}^{n-1}\left(\frac{\left(f^{-1}\right)^{(j)}(x)}{j !}\right)^{k_{j}}\right)=(-1)^{\sum_{j=1}^{n-1} j k_{j}}=(-1)^{n}
$$

for every summand of the formula in Lemma A.2. Thus $\operatorname{sign}\left(\left(f^{-1}\right)^{(n)}(x)\right)=(-1)^{n-1}$ for all $x>0$ and $n \geq 3$ showing that $f^{-1}$ is a Bernstein function. 
We want to apply Lemma 4.1 to the function

$$
\zeta(x)=\psi(-i x)=\int_{-\infty}^{0}\left(e^{x y}-1-x y\right) \mathrm{d} \phi(y)>0 \quad \text { for } x>0,
$$

where $\phi$ is the semistable Lévy measure for $\alpha \in(1,2)$ from (1.8) concentrated on the negative axis and $\psi$ is the corresponding log-characteristic function from (1.9). Note that $\zeta(x) \rightarrow \infty$ as $x \rightarrow \infty$ due to the tail behavior in (1.8) and by dominated convergence we have

$$
\zeta^{\prime}(x)=\int_{-\infty}^{0} y\left(e^{x y}-1\right) \mathrm{d} \phi(y)=\int_{0}^{\infty}\left(1-e^{-x y}\right) y \mathrm{~d} \phi(-y) .
$$

Since $\phi$ integrates $\min \left\{1, y^{2}\right\}$ and fulfills (1.8), the measure $\mu$ with $\mathrm{d} \mu(y)=y \mathrm{~d} \phi(-y)$ integrates $\min \{1, y\}$ and thus $\zeta^{\prime}$ is a Bernstein function. This also follows from the higher order derivatives

$$
\zeta^{(n)}(x)=\int_{-\infty}^{0} y^{n} e^{x y} \mathrm{~d} \phi(y) \begin{cases}>0 & \text { if } n \geq 2 \text { is even, } \\ <0 & \text { if } n \geq 3 \text { is odd }\end{cases}
$$

which additionally shows that these derivatives do not vanish. Thus from Lemma 4.1 we conclude that the inverse $\zeta^{-1}$ is a Bernstein function. This will be the key to solve an open problem concerning the following result on space-time duality from [12].

According to [30,Example 28.2], the semistable Lévy process $\left(X_{t}\right)_{t \geq 0}$ with $P\left\{X_{t} \in\right.$ $A\}=v^{* t}(A)$ for Borel sets $A \in \mathcal{B}(\mathbb{R})$, where $v$ is the semistable distribution with log-characteristic function $\psi$ from (1.9), possesses $C^{\infty}(\mathbb{R})$-densities $x \mapsto p(x, t)$ for every $t>0$ with $P\left\{X_{t} \in A\right\}=\int_{A} p(x, t) \mathrm{d} x$ and for $t=0$ we may write $p(x, 0)=\delta(x)$ corresponding to $X_{0}=0$ almost surely. It is shown in [13] that these densities are the point source solution to the semi-fractional diffusion equation

$$
\frac{\partial^{\alpha}}{\partial_{c, \theta}(-x)^{\alpha}} p(x, t)=\frac{\partial}{\partial t} p(x, t)
$$

with the negative semi-fractional derivative of order $\alpha$ from (1.14) acting on the space variable. Since the semi-fractional derivative is a non-local operator, this equation is hard to interpret from a physical point of view, whereas non-locality in time may correspond to long memory effects [10]. As a generalization of a space-time duality result for fractional diffusions $[4,11]$ based on a corresponding result for stable densities by Zolotarev [39, 40], it was shown in [12] that space-time duality may also hold for semi-fractional diffusions. Theorem 3.3 in [12] states that for $x>0$ and $t>0$ we have $p(x, t)=\alpha^{-1} h(x, t)$, where $h(x, t)$ is the point source solution to the semi-fractional differential equation

$$
\frac{\partial^{1 / \alpha}}{\partial_{\mathrm{d}, \tau} t^{1 / \alpha}} h(x, t)+\frac{\partial}{\partial x} h(x, t)=t^{-1 / \alpha} \varrho(\log t) \delta(x)
$$


with a semi-fractional derivative of order $1 / \alpha$ acting on the time variable, provided that $\tau$ and $\varrho$ are admissible functions with respect to $1 / \alpha \in\left(\frac{1}{2}, 1\right)$ and $d=c^{1 / \alpha}>1$ which remained an open problem in [12]. We will now show that indeed $\tau$ is admissible and $\varrho(x)=-\alpha \tau^{\prime}(x)$, provided that $\tau$ is smooth. Note that in general $\varrho$ will not be admissible as conjectured in [12], but we will justify the inhomogeneity in (4.3) by different arguments. In [12] the function $\tau$ appears in the following way. The above inverse $\zeta^{-1}$ is called $\xi$ in [12] and its existence is shown in Lemma 4.1 of [12]. It was further shown in Lemma 4.2 of [12] that $\xi(t)=t^{1 / \alpha} g(\log t)$ for a continuously differentiable and $\log (c)$-periodic function $g$. Since we now know that $\xi=\zeta^{-1}$ is a Bernstein function, in fact $g$ is a $C^{\infty}(\mathbb{R})$-function. Since $g$ is a smooth $\log (c)$-periodic function, it is representable by its Fourier series

$$
g(x)=\sum_{n \in \mathbb{Z}} d_{n} e^{-i n \tilde{d} x} \quad \text { with } \tilde{d}=\frac{2 \pi}{\log c}=\frac{2 \pi \frac{1}{\alpha}}{\log d} \text { for } d=c^{1 / \alpha},
$$

where by Lemma 1 in $\S 12$ of [2] we have $\left|d_{n}\right| \leq C \cdot e^{-\frac{\pi}{2}|n| \tilde{d}}$ for some $C>0$ and all $n \in \mathbb{Z}$. If we require a little more quality, namely that the Fourier coefficients even decay as

$$
\left|d_{n}\right| \leq C \cdot e^{-\frac{\pi}{2}|n| \tilde{d}_{1}}|n|^{-\frac{3}{2}-\frac{1}{\alpha}-\varepsilon} \quad \text { for some } \varepsilon>0 \text { and all } n \in \mathbb{Z} \backslash\{0\},
$$

then we can define $\tau$ by the Fourier series

$$
\tau(x)=\sum_{n \in \mathbb{Z}} \frac{d_{n}}{\Gamma\left(i n \tilde{d}-\frac{1}{\alpha}+1\right)} e^{-i n \tilde{d} x} .
$$

Lemma 4.2 If (4.5) holds, then the function $\tau$ in (4.6) is well-defined and a smooth admissible function with respect to $1 / \alpha \in\left(\frac{1}{2}, 1\right)$ and $d=c^{1 / \alpha}$. Moreover, $\zeta^{-1}$ is a self-similar Bernstein function with respect to the same parameters.

Proof As shown above $\xi=\zeta^{-1}$ is a Bernstein function and with $d=c^{1 / \alpha}$ and $\xi(t)=t^{1 / \alpha} g(\log t)$ for a $\log (c)$-periodic function $g$ we get

$$
d \cdot \xi(t)=(c t)^{1 / \alpha} g(\log (c t))=\xi(c t)=\xi\left(d^{\alpha} t\right)
$$

showing that $\xi$ is a self-similar Bernstein function with respect to $1 / \alpha \in\left(\frac{1}{2}, 1\right)$ and $d=c^{1 / \alpha}$. By Lemma 2.2 we have

$$
\xi(x)=\int_{0}^{\infty}\left(1-e^{-x y}\right) \mathrm{d} \mu(y),
$$

where $\mu$ is a semistable Lévy measure with $\mu(t, \infty)=t^{-1 / \alpha} \tau(\log t)$ for an admissible function $\tau$ with respect to $1 / \alpha \in\left(\frac{1}{2}, 1\right)$ and $d=c^{1 / \alpha}$. It remains to show that $\tau$ is 
indeed the function we are looking for. Let us assume for a moment that $\tau$ is smooth and thus admits the Fourier series

$$
\tau(x)=\sum_{n \in \mathbb{Z}} a_{n} e^{-i n \tilde{d} x} \quad \text { with } \tilde{d}=\frac{2 \pi}{\log c}=\frac{2 \pi \frac{1}{\alpha}}{\log d} .
$$

In this case the function $\gamma$ appearing in Lemma 2.2 fulfills

$$
\gamma(-x)=e^{-\frac{1}{\alpha} x} \xi\left(e^{x}\right)=g(x)
$$

and by (2.6) has the Fourier series representation

$$
g(x)=\gamma(-x)=\sum_{n \in \mathbb{Z}} a_{n} \Gamma\left(i n \tilde{d}-\frac{1}{\alpha}+1\right) e^{-i n \tilde{d} x} .
$$

A comparison with (4.4) and uniqueness of the Fourier coefficients shows that $a_{n}$ coincides with $d_{n} / \Gamma\left(\right.$ in $\left.\tilde{d}-\frac{1}{\alpha}+1\right)$ and thus $\tau$ is indeed the function in (4.6). Finally, we have to show that the series in (4.6) converges. Using the asymptotic behavior of the gamma function in Corollary 1.4.4 of [1], the Fourier coefficients fulfill

$$
\left|\frac{d_{n}}{\Gamma\left(i n \tilde{d}-\frac{1}{\alpha}+1\right)}\right| \leq K\left|d_{n}\right| \cdot|n|^{-\frac{1}{2}+\frac{1}{\alpha}} e^{\frac{\pi}{2}|n| \tilde{d}}
$$

for a constant $K>0$ and all $n \in \mathbb{Z} \backslash\{0\}$. According to our assumption (4.5) we obtain

$$
\left|\frac{d_{n}}{\Gamma\left(i n \tilde{d}-\frac{1}{\alpha}+1\right)}\right| \leq K C|n|^{-2-\varepsilon}
$$

for some $\varepsilon>0$ and all $n \in \mathbb{Z} \backslash\{0\}$ showing that the series in (4.6) converges and the resulting function is continuously differentiable by Theorem 2.6 in [8].

Admissibility of $\tau$ in combination with Theorem 3.3 in [12] finally enables us to completely solve space-time duality for semi-fractional diffusions. The Eq. (4.3) is derived in [12] by Laplace inversion of the equation

$$
\xi(s) \tilde{h}(x, s)-\frac{1}{s} \xi(s) \delta(x)+\frac{\partial}{\partial x} \tilde{h}(x, s)=-\frac{1}{s} \frac{f(s)}{s+f(s)} \xi(s) \delta(x),
$$

where $f$ is defined in the proof of Theorem 3.1 in [12] as

$$
f(s)=\frac{1}{\alpha} \xi(s)^{\alpha} m^{\prime}(\log \xi(s))
$$

and $m$ is a $\log \left(c^{1 / \alpha}\right)$-periodic function given by

$$
\zeta(s)=x^{\alpha} m(\log x)
$$


Theorem 4.3 Assume that (4.5) holds, $\tau$ is given as in Lemma 4.2 and define $\varrho(t)=-\alpha \tau^{\prime}(t)$. Then the point source solutions $p(x, t)$ of the semi-fractional diffusion Eq. (4.2) of order $\alpha \in(1,2)$ in space and $h(x, t)$ of the semi-fractional Eq. (4.3) of order $1 / \alpha \in\left(\frac{1}{2}, 1\right)$ in time are equivalent, i.e. $p(x, t)=\alpha^{-1} h(x, t)$ for all $x>0$ and $t>0$.

Proof As shown in [12] it remains to carry out Laplace inversion of (4.8). Clearly, $\frac{\partial}{\partial x} \widetilde{h}(x, s)$ is the Laplace transform of $\frac{\partial}{\partial x} h(x, t)$. Moreover, the first part on the lefthand side of (4.8) is the Laplace transform of the semi-fractional derivative in time $\frac{\partial^{1 / \alpha}}{\partial_{d, \tau} \tau^{1 / \alpha}} h(x, t)$ as argued in [12]. Since both derivatives are continuous functions of the time variable, Laplace inversion of the left-hand side of (4.8) yields the sum of these derivatives.

We may rewrite the right-hand side of (4.8) as follows. From (4.10) we obtain

$$
\zeta^{\prime}(t)=\alpha x^{\alpha-1} m(\log x)+x^{\alpha-1} m^{\prime}(\log x)
$$

and thus we have by (4.10) and $\zeta(\xi(s))=s$

$$
\zeta^{\prime}(\xi(s))=\alpha \frac{1}{\xi(s)} s+\frac{1}{\xi(s)} \xi(s)^{\alpha} m^{\prime}(\log \xi(s))=\alpha \frac{1}{\xi(s)} s+\alpha \frac{1}{\xi(s)} f(s),
$$

where the last equality follows from (4.9). This shows that

$$
f(s)=\frac{1}{\alpha} \zeta^{\prime}(\xi(s)) \xi(s)-s
$$

and the right-hand side of (4.8) can be rewritten as

$$
-\frac{1}{s} \frac{f(s)}{s+f(s)} \xi(s) \delta(x)=-\frac{\alpha}{s} \frac{f(s)}{\zeta^{\prime}(\xi(s))} \delta(x)=-\frac{1}{s} \xi(s) \delta(x)+\alpha \frac{1}{\zeta^{\prime}(\xi(s))} \delta(x) .
$$

Note that the first part $-\frac{1}{s} \xi(s) \delta(x)$ also appears on the left-hand side of (4.8); cf. Remark 4.4. Moreover, since $\frac{1}{s}$ is the Laplace transform of $1_{(0, \infty)}(t)$, the function $\frac{1}{s} \xi(s)$ is the Laplace transform of the Riemann-Liouville semi-fractional derivative of $1_{(0, \infty)}(t)$ which is

$$
\frac{\mathrm{d}}{\mathrm{d} t} \int_{0}^{t} 1_{(0, \infty)}(t-s) s^{-1 / \alpha} \tau(\log (s)) \mathrm{d} s=t^{-1 / \alpha} \tau(\log (t))
$$

Now from $\zeta(\xi(s))=s$ we get $\zeta^{\prime}(\xi(s)) \cdot \xi^{\prime}(s)=1$ and hence it follows from (4.7) and integration by parts

$$
\begin{aligned}
\frac{1}{\zeta^{\prime}(\xi(s))} & =\xi^{\prime}(s)=\int_{0}^{\infty} e^{-s t} t \mathrm{~d} \mu(t) \\
& =\left[-e^{-s t} t^{1-1 / \alpha} \tau(\log t)\right]_{t=0}^{\infty}+\int_{0}^{\infty}\left(-s e^{-s t} t+e^{-s t}\right) t^{-1 / \alpha} \tau(\log t) \mathrm{d} t
\end{aligned}
$$




$$
=-s \int_{0}^{\infty} e^{-s t} t^{1-1 / \alpha} \tau(\log t) \mathrm{d} t+\int_{0}^{\infty} e^{-s t} t^{-1 / \alpha} \tau(\log t) \mathrm{d} t
$$

which is the Laplace transform of

$$
\begin{aligned}
- & \frac{\mathrm{d}}{\mathrm{d} t}\left(t^{1-1 / \alpha} \tau(\log t)\right)+t^{-1 / \alpha} \tau(\log t) \\
& =-\left(1-\frac{1}{\alpha}\right) t^{-1 / \alpha} \tau(\log t)-t^{-1 / \alpha} \tau^{\prime}(\log t)+t^{-1 / \alpha} \tau(\log t) \\
& =t^{-1 / \alpha}\left(\frac{1}{\alpha} \tau(\log t)-\tau^{\prime}(\log t)\right) .
\end{aligned}
$$

Putting things together, due to continuous differentiability of $\tau$ proven in Lemma 4.2, Laplace inversion of the right-hand side of (4.8) yields

$$
\left(-t^{-1 / \alpha} \tau(\log t)+\alpha t^{-1 / \alpha}\left(\frac{1}{\alpha} \tau(\log t)-\tau^{\prime}(\log t)\right)\right) \delta(x)=-\alpha t^{-1 / \alpha} \tau^{\prime}(\log t) \delta(x)
$$

concluding the proof.

Remark 4.4 Note that in the stable case we have that $\tau$ is constant and thus $\tau^{\prime} \equiv 0$. Hence we recover space-time duality for fractional diffusions in [11] as a special case. Further note that with (4.11) the term $-\frac{1}{s} \xi(s) \delta(x)$ appears on both sides of (4.8) and can be cancelled. After cancellation the first part on the left-hand side of (4.8) is the Laplace transform of the Riemann-Liouville semi-fractional derivative of $h(x, t)$ and as in the proof of Theorem 4.3 we may rewrite (4.3) as

$$
\mathbb{D}_{d, \tau}^{1 / \alpha} h(x, t)+\frac{\partial}{\partial x} h(x, t)=t^{-1 / \alpha}\left(\tau(\log t)-\alpha \tau^{\prime}(\log t)\right) \delta(x),
$$

where the semi-fractional derivative acts on the time variable and now the inhomogeniety on the right-hand side is non-negative by Lemma A.1.

Acknowledgements We are grateful to an anonymous referee for several useful comments leading to an improvement of the presentation.

Funding Open Access funding enabled and organized by Projekt DEAL. The authors have no relevant financial or non-financial interests to disclose. The authors did not receive support from any organization for the submitted work.

Data Availability Data sharing is not applicable to this article as no datasets were generated or analyzed during the current study.

Open Access This article is licensed under a Creative Commons Attribution 4.0 International License, which permits use, sharing, adaptation, distribution and reproduction in any medium or format, as long as you give appropriate credit to the original author(s) and the source, provide a link to the Creative Commons licence, and indicate if changes were made. The images or other third party material in this article are included in the article's Creative Commons licence, unless indicated otherwise in a credit line to the material. If material is not included in the article's Creative Commons licence and your intended use is not permitted by statutory regulation or exceeds the permitted use, you will need to obtain permission directly from the copyright holder. To view a copy of this licence, visit http://creativecommons.org/licenses/by/4.0/. 


\section{Appendix}

We first show an elementary result connecting admissible functions with the representation in Corollary 7.4.4 of [21].

Lemma A.1 Let $\theta: \mathbb{R} \rightarrow(0, \infty)$ be a periodic function and $\alpha>0$. Then the following statements are equivalent.

(i) $\theta(y+\delta) \leq e^{\alpha \delta} \theta(y)$ for all $y>0$ and $\delta>0$.

(ii) $\theta(y+\delta) \leq e^{\alpha \delta} \theta(y)$ for all $y \in \mathbb{R}$ and $\delta \geq 0$.

(iii) $\theta(y-\delta) \geq e^{-\alpha \delta} \theta(y)$ for all $y \in \mathbb{R}$ and $\delta \geq 0$.

(iv) The mapping $t \mapsto t^{-\alpha} \theta(\log t)$ is non-increasing for $t>0$.

If $\theta$ is additionally differentiable, then each statement (i)-(iv) is equivalent to

(v) $\theta^{\prime}(y) \leq \alpha \theta(y)$ for all $y \in \mathbb{R}$.

Proof "(i) $\Rightarrow$ (ii)": Note that (ii) is trivially true for $\delta=0$. If $\theta$ has period $p>0$ and $y \leq 0$, choose $k \in \mathbb{N}$ such that $y+k p>0$. Then $\theta(y+\delta)=\theta(y+k p+\delta) \leq$ $e^{\alpha \bar{\delta}} \theta(y+k p)=e^{\alpha \delta} \theta(y)$.

"(ii) $\Rightarrow$ (iii)": Write $z=y-\delta$ then $\theta(y-\delta)=\theta(z) \geq e^{-\alpha \delta} \theta(z+\delta)=e^{-\alpha \delta} \theta(y)$.

"(iii) $\Rightarrow$ (iv)": For $0<s<t$ write $t=e^{y}$ and $s=e^{y-\delta}$ for some $y \in \mathbb{R}$ and $\delta>0$.

Then $s^{-\alpha} \theta(\log s)=e^{-\alpha(y-\delta)} \theta(y-\delta) \geq e^{-\alpha y} \theta(y)=t^{-\alpha} \theta(\log t)$.

"(iv) $\Rightarrow$ (i)": Write $y=\log t$ and $\delta=\log \gamma$ for some $t, \gamma>1$. Then $\theta(y+\delta)=$ $\theta(\log (\gamma t))=(\gamma t)^{\alpha}(\gamma t)^{-\alpha} \theta(\log (\gamma t)) \leq(\gamma t)^{\alpha} t^{-\alpha} \theta(\log t)=e^{\alpha \delta} \theta(y)$.

"(ii) $\Rightarrow(\mathrm{v})$ ": For fixed $y \in \mathbb{R}$ we have $\frac{\theta(y+\delta)-\theta(y)}{\delta} \leq \frac{e^{\alpha \delta}-1}{\delta} \theta(y)$ for all $\delta>0$ and for differentiable $\theta$ as $\delta \downarrow 0$ we get $\theta^{\prime}(y) \leq \alpha \theta(y)$.

“(v) $\Rightarrow($ iv $) ":$ We have $\frac{d}{d t}\left(t^{-\alpha} \theta(\log t)\right)=t^{-\alpha-1}\left(-\alpha \theta(\log t)+\theta^{\prime}(\log t)\right) \leq 0$.

Now we derive a formula for higher order derivatives of inverse functions used in Sect. 3 for which we couldn't find a suitable reference.

Lemma A.2 Let $A, B \subseteq \mathbb{R}$ be open and let $f: A \rightarrow B$ be an invertible $C^{n}(A)$ function for some $n \in \mathbb{N}$ such that $f^{\prime}(x) \neq 0$ for all $x \in A$. Then $f^{-1}: B \rightarrow A$ is of class $C^{n}(B)$ and for $n \geq 2$ we have

$$
\begin{aligned}
&\left(f^{-1}\right)^{(n)}(x)=-\frac{1}{f^{\prime}\left(f^{-1}(x)\right)}{ }_{k_{1}+2 k_{2}+\cdots+(n-1) k_{n-1}=n} \frac{n !}{k_{1} ! \cdots k_{n-1} !} f^{\left(k_{1}+\cdots+k_{n-1}\right)}\left(f^{-1}(x)\right) \\
& \cdot \prod_{j=1}^{n-1}\left(\frac{\left(f^{-1}\right)^{(j)}(x)}{j !}\right)^{k_{j}} \cdot
\end{aligned}
$$

Remark A.3 For $n=1$ it is well known that $\left(f^{-1}\right)^{\prime}(x)=\frac{1}{f^{\prime}\left(f^{-1}(x)\right)}$. For $n=2$ we have $k_{1}=2$ in Lemma A.3 so that

$$
\left(f^{-1}\right)^{\prime \prime}(x)=-\frac{1}{f^{\prime}\left(f^{-1}(x)\right)} f^{\prime \prime}\left(f^{-1}(x)\right) \cdot\left(\left(f^{-1}\right)^{\prime}(x)\right)^{2}=-\frac{f^{\prime \prime}\left(f^{-1}(x)\right)}{\left(f^{\prime}\left(f^{-1}(x)\right)\right)^{3}} .
$$


Iterating this procedure leads to a formula for $\left(f^{-1}\right)^{(n)}$ given in [38] not involving the derivatives of lower order $\left(f^{-1}\right)^{\prime}, \ldots,\left(f^{-1}\right)^{(n-1)}$.

Proof of Lemma A.2 We use Faà di Bruno's formula of higher order chain rule

$$
\frac{\mathrm{d}^{n}}{\mathrm{~d} x^{n}} f(g(x))=\sum_{k_{1}+2 k_{2}+\cdots+n k_{n}=n} \frac{n !}{k_{1} ! \cdots k_{n} !} f^{\left(k_{1}+\cdots+k_{n}\right)}(g(x)) \prod_{j=1}^{n}\left(\frac{\left(g^{(j)}(x)\right.}{j !}\right)^{k_{j}} .
$$

For $j=n$ we must have $k_{n}=1$ and $k_{1}=\cdots=k_{n-1}=0$, otherwise $k_{n}=0$ if $k_{j} \geq 1$ for some $j \in\{1, \ldots, n-1\}$. Hence we get

$$
\begin{aligned}
& \frac{\mathrm{d}^{n}}{\mathrm{~d} x^{n}} f(g(x))=\sum_{k_{1}+2 k_{2}+\cdots+(n-1) k_{n-1}=n} \frac{n !}{k_{1} ! \cdots k_{n} !} f^{\left(k_{1}+\cdots+k_{n-1}\right)}(g(x)) \prod_{j=1}^{n-1}\left(\frac{\left(g^{(j)}(x)\right.}{j !}\right)^{k_{j}} \\
& +f^{\prime}(g(x)) g^{(n)}(x) .
\end{aligned}
$$

If $g=f^{-1}$, we know that $\frac{\mathrm{d}^{n}}{\mathrm{~d} x^{n}} f\left(f^{-1}(x)\right)=0$ for $n \geq 2$ which directly leads to the stated formula for $\left(f^{-1}\right)^{(n)}$.

\section{References}

1. Andrews, G.E., Askey, R., Roy, R.: Special Functions. Cambridge University Press, Cambridge (1999)

2. Arnold, V.I.: Geometrical Methods in the Theory of Ordinary Differential Equations, 2nd edn. Springer, New York (1983)

3. Ascione, G.: Abstract Cauchy problems for the generalized fractional calculus. Nonlinear Anal. 209, 112339 (2021)

4. Baeumer, B., Meerschaert, M.M., Nane, E.: Space-time duality for fractional diffusion. J. Appl. Probab. 46, 110-115 (2009)

5. Bouzar, N.: The semi-Sibuya distribution. Ann. Inst. Stat. Math. 60, 459-464 (2008)

6. Christoph, G., Schreiber, K.: Scaled Sibuya distribution and discrete self-decomposability. Statist. Probab. Lett. 48, 181-187 (2000)

7. Feller, W.: An Introduction to Probability Theory and Its Applications, vol. II, 2nd edn. Wiley, New York (1971)

8. Folland, G.B.: Fourier Analysis and Its Applications. Wadsworth, Belmont (1992)

9. Hanyga, A.: A comment on a controversial issue: a generalized fractional derivative cannot have a regular kernel. Fract. Calc. Appl. Anal. 23(1), 211-223 (2020)

10. Hilfer, R.: Threefold introduction to fractional derivatives. In: Klages, R., et al. (eds.) Anomalous Transport: Foundations and Applications, pp. 17-74. Wiley-VCH, Weinheim (2008)

11. Kelly, J.F., Meerschaert, M.M.: Space-time duality for the fractional advection-dispersion equation. Water Resour. Res. 53, 3464-3475 (2017)

12. Kern, P., Lage, S.: Space-time duality for semi-fractional diffusions. In: Freiberg, U., et al. (eds.) Fractal Geometry and Stochastics VI: Progress in Probability, vol. 76, pp. 255-272. Basel, Birkhäuser (2021)

13. Kern, P., Lage, S., Meerschaert, M.M.: Semi-fractional diffusion equations. Fract. Calc. Appl. Anal. 22(2), 326-357 (2019)

14. Kern, P., Meerschaert, M.M., Xiao, Y.: Asymptotic behavior of semistable Lévy exponents and applications to fractal path properties. J. Theor. Probab. 31, 598-617 (2018)

15. Kevei, P.: Regularly log-periodic functions and some applications. Probab. Math. Stat. 40(1), 159-182 (2020)

16. Kilbas, A.A., Srivastava, H.M., Trujillo, J.J.: Theory and Applications of Fractional Differential Equations. North-Holland Mathematical Studies, vol. 204, Elsevier, Amsterdam (2006) 
17. Kochubei, A.N.: General fractional calculus, evolution equations, and renewal processes. Integr. Equ. Oper. Theory 71, 583-600 (2011)

18. Kochubei, A.N., Kondratiev, Y., da Silva, J.L.: From random times to fractional kinetics. Interdiscip. Stud. Complex Syst. 16, 5-32 (2020)

19. Kozubowski, T.J., Podgórski, K.: A generalized Sibuya distribution. Ann. Inst. Stat. Math. 70, 855-887 (2018)

20. Liu, W., Röckner, M., da Silva, J.L.: Strong dissipativity of generalized time-fractional derivatives and quasi-linear (stochastic) partial differential equations. J. Funct. Anal. 281(8), 109135 (2021)

21. Meerschaert, M.M., Scheffler, H.P.: Limit Distributions for Sums of Independent Random Vectors. Wiley, New York (2001)

22. Meerschaert, M.M., Sikorskii, A.: Stochastic Models for Fractional Calculus. De Gruyter, Berlin (2012)

23. Nichols, J.A., Henry, B.I., Angstmann, C.N.: Subdiffusive discrete time random walks via Monte Carlo and subordination. J. Comput. Phys. 372, 373-384 (2018)

24. Pachon, A., Polito, F., Ricciuti, C.: On discrete-time semi-Markov processes. Discrete Cont. Dyn. Syst. B 26(3), 1499-1529 (2021)

25. Patie, P., Srapionyan, A.: Self-similar Cauchy problems and generalized Mittag-Leffler functions. Fract. Calc. Appl. Anal. 24(2), 447-482 (2021)

26. Podlubny, I.: Fractional Differential Equations. Academic Press, San Diego (1998)

27. Samko, S.G., Cardoso, R.P.: Integral equations of the first kind of Sonine type. Int. J. Math. Math. Sci. 57, 3609-3632 (2003)

28. Samko, S.G., Kilbas, A.A., Marichev, O.I.: Fractional Integrals and Derivatives. Gordon and Breach, London (1993)

29. Sandev, T., Metzler, R., Chechkin, A.: From continuous time random walks to the generalized diffusion equation. Fract. Calc. Appl. Anal. 21(1), 10-28 (2018)

30. Sato, K.: Lévy Processes and Infinitely Divisible Distributions. Cambridge University Press, Cambridge (1999)

31. Schilling, R.L., Song, R., Vondraček, Z.: Bernstein Functions, 2nd edn. De Gruyter, Berlin (2012)

32. Sibuya, M.: Generalized hypergeometric, digamma, and trigamma distributions. Ann. Inst. Stat. Math. 33, 177-190 (1979)

33. Sonine, N.: Sur la généralisation d'une formule d'Abel. Acta Math. 4, 171-176 (1884)

34. Sornette, D.: Discrete-scale invariance and complex dimensions. Phys. Rep. 297, 239-270 (1998)

35. Sornette, D.: Critical Phenomena in Natural Sciences. Springer, Berlin (2000)

36. Toaldo, B.: Convolution-type derivatives, hitting-times of subordinators and time-changed $C_{0^{-}}$ semigroups. Potential Anal. 42, 115-140 (2015)

37. Wick, J.: Über eine Integralgleichung vom Abelschen Typ. Z. Angew. Math. Mech. 48(8), T39-T41 (1968)

38. Zabrěko, P.P., Lysenko, Y.V.: Exact formulas for higher-order derivatives of inverse functions in Banach spaces (in Russian). Dokl. Nats. Akad. Nauk Belarusi 45(2), 27-30 (2001)

39. Zolotarev, V.M.: Expressions of the density of a stable distribution with exponent $\alpha$ greater than one by means of a frequency with exponent $1 / \alpha$. In: Selected Translations in Mathematical Statistics and Probability, vol. 1, AMS, Providence, pp. 163-167 (1961)

40. Zolotarev, V.M.: One-Dimensional Stable Distributions. Translations of Mathematical Monographs, vol. 65, AMS, Providence (1986)

Publisher's Note Springer Nature remains neutral with regard to jurisdictional claims in published maps and institutional affiliations. 